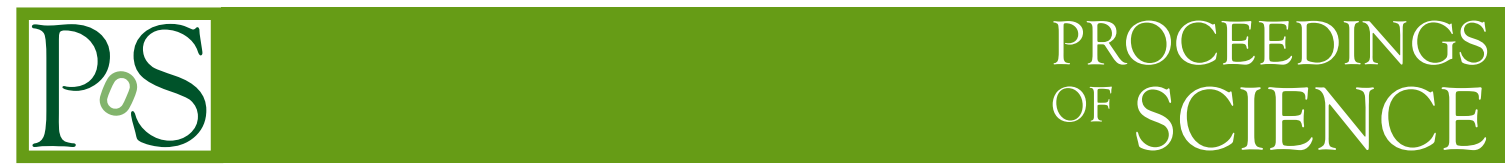

\title{
Jet production measurements at CMS
}

\section{Panagiotis KOKKAS ${ }^{* i}$}

University of Ioannina

E-mail: pkokkaseuoi.gr

\begin{abstract}
Measurements on inclusive jet and di-jet cross sections, 3-jet mass cross sections, event shapes and studies on color coherence effects are presented. The data samples used for these measurements were collected during 2011 and 2012 at a proton-proton centre-of-mass energy of 7 and 8 $\mathrm{TeV}$, respectively, with the CMS detector at the LHC. The measurements on inclusive jet, di-jet and 3-jet mass cross sections are compared to the predictions from perturbative QCD at nextto-leading order theoretical calculations. Within uncertainties, data and theory are in agreement. Event shape and color coherence measurements are compared also to various Monte Carlo event generators providing useful information for further tuning of these generators.
\end{abstract}

XXII. International Workshop on Deep-Inelastic Scattering and Related Subjects, 28 April - 2 May 2014

Warsaw, Poland

\footnotetext{
* Speaker.

${ }^{\dagger}$ For the CMS Collaboration.
} 


\section{Introduction}

Jet measurements at Large Hadron Collider (LHC) are of great importance because they provide a test of perturbative Quantum Chromodynamics (pQCD) in a previously unexplored energy region. They provide a precise measurement of the main background for many of the new physics searches and test the Standard Model (SM) predictions at high energy scales.

In these proceedings measurements on inclusive jet and di-jet cross sections, 3-jet mass cross sections, event shapes and studies on color coherence effects are presented, using data collected with the Compact Muon Solenoid (CMS) detector [1] at the CERN LHC during the 2011 run at $\sqrt{s}$ $=7 \mathrm{TeV}$ and the 2012 run at $\sqrt{s}=8 \mathrm{TeV}$.

\section{The measurements}

CMS has delivered the measurement of the inclusive jet production cross section as a function of the jet $p_{\mathrm{T}}$ and rapidity, using data collected at $7 \mathrm{TeV}$ [2] and at $8 \mathrm{TeV}$ [3]. Figure 1], on the left, shows the comparison of the inclusive jet production cross section at $8 \mathrm{TeV}$ to next-to-leading order (NLO) predictions using the NNPDF2.1 PDF set [4] times the non-perturbative (NP) correction factor. The measurement in the low $p_{\mathrm{T}}$ region $(21-74 \mathrm{GeV})$ is done in seven rapidity bins up to $|y|=4.7$ while in the high $p_{\mathrm{T}}$ region $(74 \mathrm{GeV}-2.5 \mathrm{TeV})$ is done in six rapidity bins up to $|y|=3.0$. It is observed that $\mathrm{pQCD}$ is able to describe the observable over two orders of magnitude in $p_{\mathrm{T}}$ and 14 orders of magnitude in the cross section. The same figure on the right presents the ratio of data over theory at NLO times NP correction for the NNPDF2.1 PDF set and for the innermost rapidity bin. For comparison predictions employing four other PDF sets (CT10 [5], MSTW2008 [6, 7], HERAPDF1.5 [8] and ABM11 [9]) are shown in addition to the total experimental systematic uncertainty. Within uncertainties most PDF sets are able to describe the data. Significant disagreements are exhibited by the ABM11 PDF set.

CMS has also delivered the measurement of the dijet production cross section as a function of the invariant mass $m_{j j}$ and the maximum rapidity $y_{\max }$ of the 2-jet system [2], using data collected at $7 \mathrm{TeV}$. The measurement is done in five rapidity bins up to $|y|=2.5$ and covers dijet masses up to $5 \mathrm{TeV}$. Figure 2 , on the left, shows the comparison of the dijet cross sections for the five different rapidity bins to the NLO prediction employing the NNPDF2.1 PDF set times NP corrections. A nice agreement is observed between data and theory. The same figure on the right presents the ratio of dijet cross sections to the theoretical prediction using the central value of the NNPDF2.1 PDF set for the inner rapidity bin. The solid histograms show the ratio of the cross sections calculated with the other PDF sets to that calculated with NNPDF2.1. Within uncertainties most PDF sets are able to describe the data.

The next measurement, reported in these proceedings, is the double-differential 3-jet mass cross section [10] as a function of the invariant mass $m_{3}$ and the maximum rapidity $y_{\max }$ of the 3 -jet system. The measurement is done in two rapidity bins with $\left|y_{\max }\right|<1$ and $1 \leq|y|_{\max }<2$ and compared to NLO theoretical calculations. Figure B, on the left, shows the comparison of the 3-jet mass distribution to the NLO prediction employing the MSTW2008-NLO PDF set times NP corrections, for the two considered regions in $|y|_{\max }$. It is observed that pQCD is able to describe the 3-jet mass cross section over five orders of magnitude and for 3-jet masses up to $3 \mathrm{TeV}$. The 

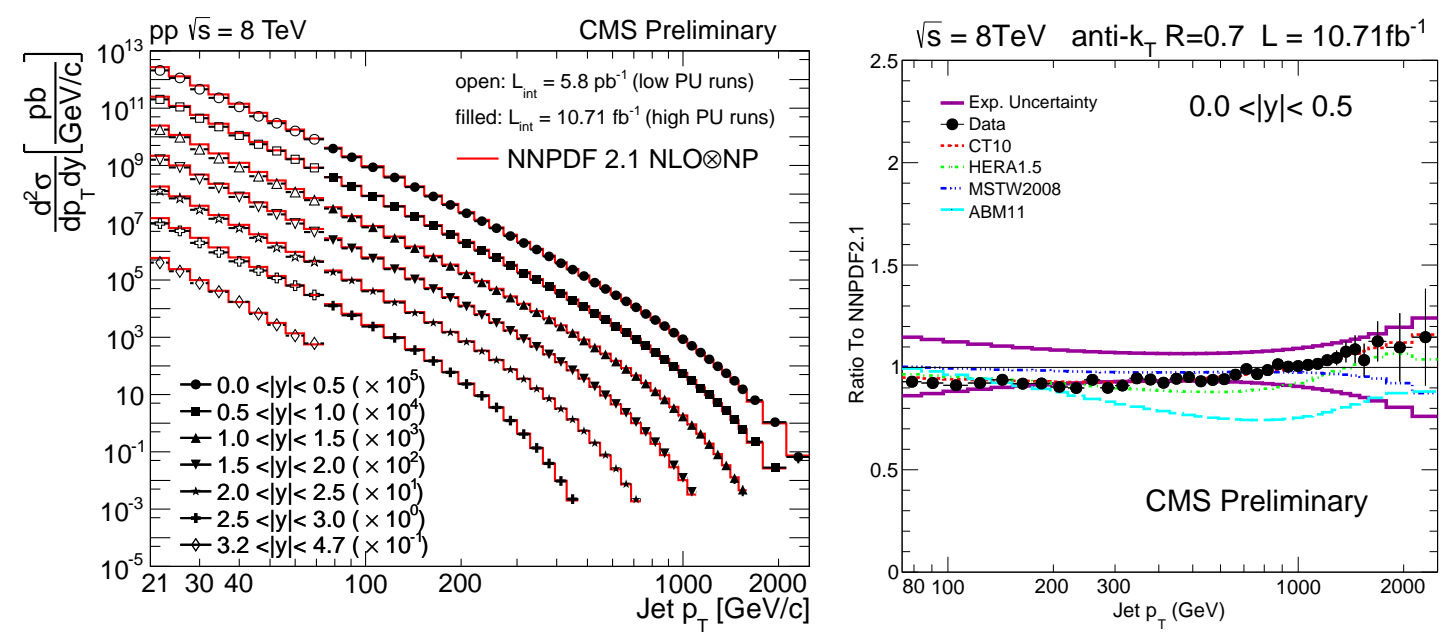

Figure 1: On the left : the double-differential inclusive jet cross section in comparison to NLO predictions using the NNPDF2.1 PDF set times the NP correction factor. On the right: the ratio of data over theory at NLO times NP correction for the NNPDF2.1 PDF set. For comparison predictions employing four other PDF sets are shown in addition to the total experimental systematic uncertainty (band enclosed by thick solid lines). The error bars correspond to the statistical uncertainty of the data.
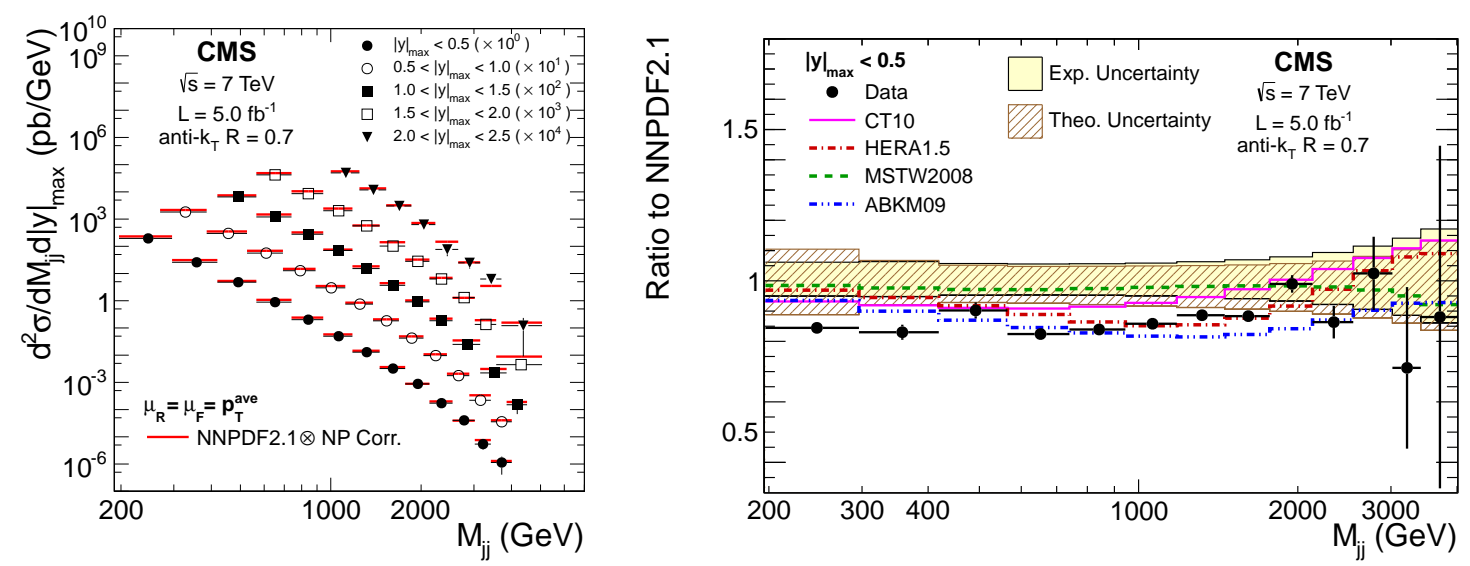

Figure 2: On the left : the dijet cross sections for the five different rapidity bins, for data (markers) and theory (thick lines) using the NNPDF2.1 PDF set. On the right: the ratio of dijet cross sections to the theoretical prediction using the central value of the NNPDF2.1 PDF set for the inner rapidity bin. The solid histograms show the ratio of the cross sections calculated with the other PDF sets to that calculated with NNPDF2.1. The experimental and theoretical systematic uncertainties are represented by the continuous and hatched bands, respectively 
same figure on the right presents the ratios of the measured cross sections for the inner rapidity bin $|y|_{\max }<1$ to theoretical predictions including NP effects, using various PDF sets with NLO PDF evolution. Results are similar also for the outer rapidity bin $1 \leq|y|_{\max }<2$. Within uncertainties most PDF sets are able to describe the data. Small deviations are visible with the HERAPDF1.5 NLO set. Significant disagreements are exhibited by the ABM11 PDF set.
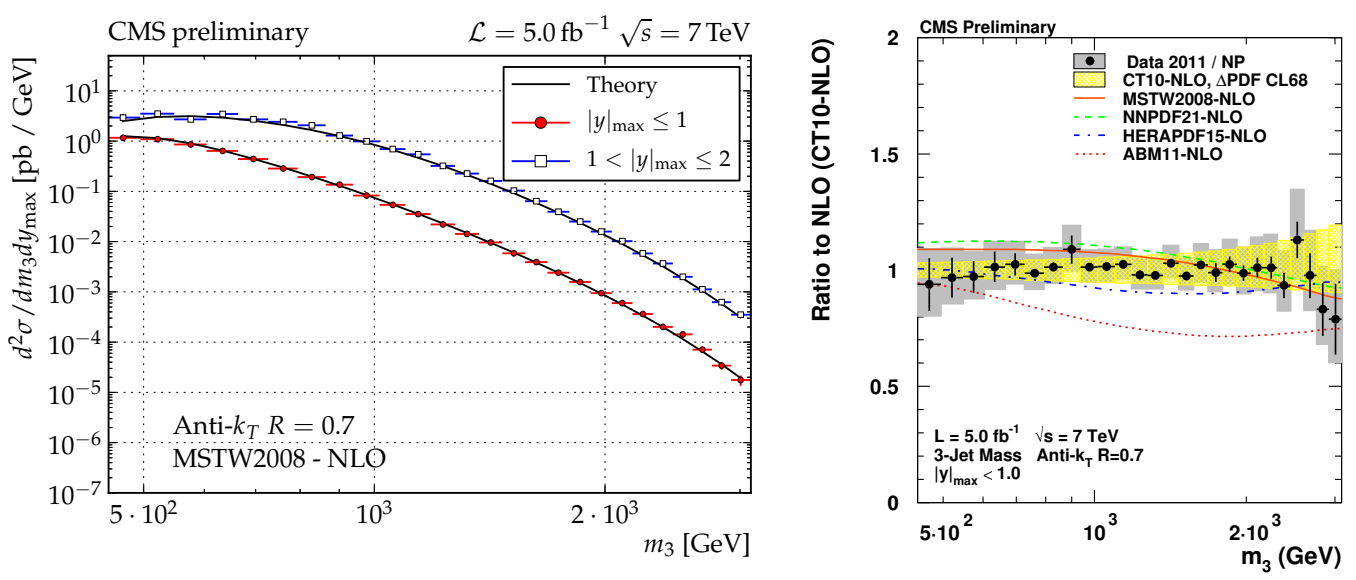

Figure 3: On the left : the comparison of the unfolded 3-jet mass cross section with the NLO prediction times NP corrections for the two considered regions in $|y|_{\max }$ using the MSTW2008-NLO PDF set. The error bars represent the total experimental uncertainty. On the right: the ratio of the unfolded 3-jet mass distribution to the theory prediction including NP effects using PDF sets with NLO PDF evolution in the inner rapidity bin $|y|_{\max }<1$. The data are shown with error bars representing the statistical uncertainty and gray squares for the systematic uncertainties. The PDF uncertainty is shown for the CT10 PDF set at 68\% confidence level as yellow band. In addition the central predictions are displayed for the other four examined PDF sets MSTW2008, NNPDF2.1, HERAPDF1.5, and ABM11.

Event shape variables are geometric properties of the energy flow in hadronic final states. They are sensitive to the details of the features of QCD. CMS has delivered measurements of several hadronic event shapes [11] using data collected at $7 \mathrm{TeV}$. These measurements can be used for tuning and validation of various QCD Monte Carlo (MC) event generators, like PYTHIA6 [12], PYTHIA8 [13], HERWIG++ [14] and MADGRAPH [15]. The most common event shape variable is the event thrust observable in the transverse plane which is sensitive to the modelling of two-jet and multi-jet topologies. Figure 4 , on the top, shows the comparison between data and various MC models of the transverse thrust in events with $250<p_{\mathrm{T}}<320 \mathrm{GeV}$ for the leading jet. The same figure on the bottom, presents the ratio of MC predictions with respect to data. All generators show an overall agreement with data within 10\%, with PYTHIA8 and HERWIG++ exhibiting a better agreement than the others.

The phenomenon called color coherence, is an important feature of the color interaction in QCD, where outgoing partons produced in the hard interaction continue to interfere with each other during their fragmentation phase. In hadron collisions, in addition to the color connection between the final-state partons, the color connection between the outgoing partons and the incoming partons must be also considered.

The study[16] is based on 3-jet events, where two leading jets exhibit a back-to-back topology. 


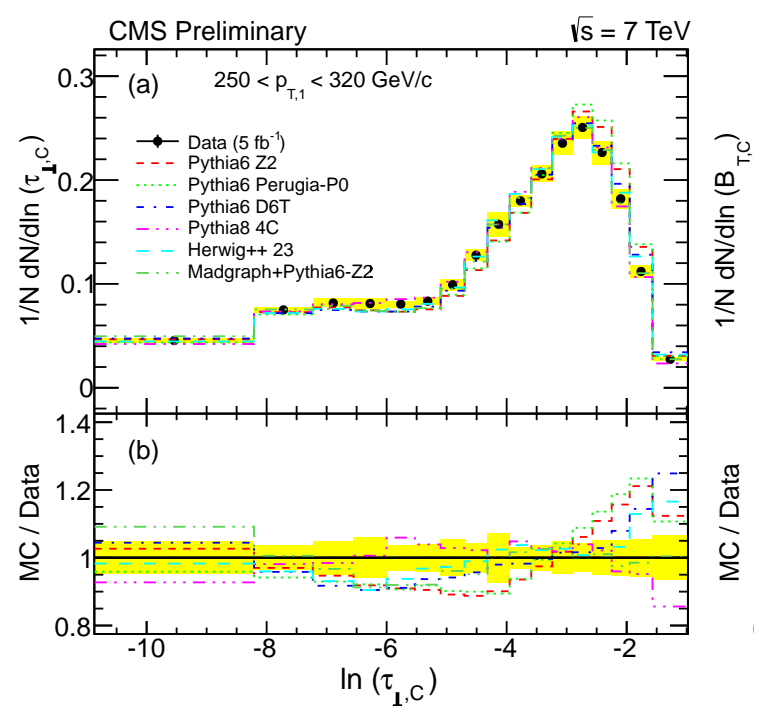

Figure 4: On the top, the comparison between data and various MC models of the transverse thrust in events with $250<p_{\mathrm{T}}<320 \mathrm{GeV} / \mathrm{c}$ for the leading jet. Bellow the ratio of MC samples with respect to data.

One of the two partons, corresponding to the two leading jets, may radiate a third parton. When color coherence effects are present the third jet, coming from the third parton, will tend to lie in the event plane defined by the emitting parton and the beam axis. The measured observable $\beta$ is defined as the azimuthal angle of the third jet with respect to the second jet in $(\eta, \phi)$ space. In the presence of color coherence, the third jet population along the event plane (in particular near $\beta \approx 0$ ) will be enhanced while out of the plane $(\beta \approx \pi / 2)$ will be suppressed.

The unfolded $\beta$ distributions are shown in Fig. 5 together with the predictions from various MC models for the central $\left(\left|\eta_{2}\right| \leq 0.8\right)$ and forward $\left(0.8<\left|\eta_{2}\right| \leq 2.5\right)$ regions. The data exhibit a clear enhancement of events compared to the various MC generators near the event plane $(\beta \approx 0)$ and a suppression in the transverse plane $(\beta \approx \pi / 2)$. None of the models used in this analysis describes data satisfactory and this measurement can be used for further tuning of the $\mathrm{MC}$ generators.

\section{Conclusions}

In this paper measurements on inclusive jet cross section, di-jet cross sections, multi-jets, event shapes and studies on color coherence effects are presented using CMS data at 7 and $8 \mathrm{TeV}$. The measurements on inclusive jet, di-jet and 3-jet mass cross sections are compared to NLO theoretical calculations using various PDF sets. Within uncertainties most PDF sets are able to describe the data. Event shape and color coherence measurements are compared to various MC event generators and provide useful information for further tuning of these generators.

\section{Acknowledgments}

This research has been co-financed by the European Union (European Social Fund - ESF) and Greek national funds through the Operational Program "Education and Lifelong Learning" of 


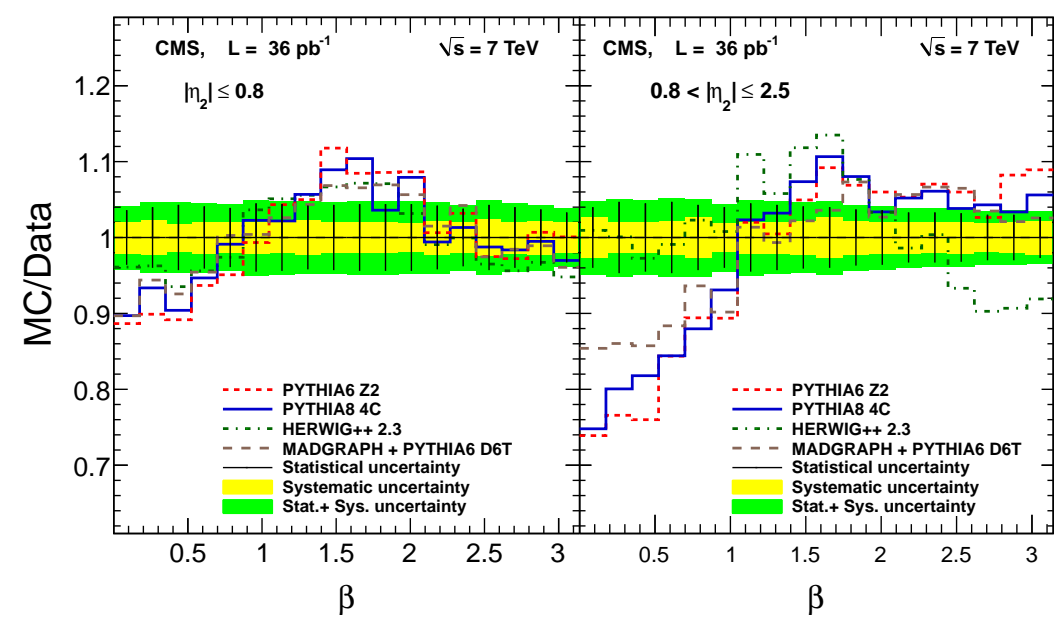

Figure 5: Observed $\beta$ distributions for the data, corrected for detector effects, and for the MC generators (PYTHiA6, PYTHIA8, HERWIG++, and MADGRAPH + PYTHiA6) in the central $\left(\left|\eta_{2}\right| \leq 0.8\right)$ and forward $\left(0.8<\left|\eta_{2}\right| \leq 2.5\right)$ regions. The error bars show the statistical uncertainties, while the yellow shaded bands correspond to the combined systematic uncertainty.

the National Strategic Reference Framework (NSRF) - Research Funding Program: ARISTEIA. Investing in knowledge society through the European Social Fund.

\section{References}

[1] CMS Collaboration, JINST 3 (2008) S08004.

[2] CMS Collaboration, PRD 87 (2013) 112002.

[3] CMS Collaboration, [CMS PAS SMP-12-012].

[4] R. D. Ball et al., Nucl. Phys. B 849 (2011) 296.

[5] H.-L. Lai et al., Phys. Rev. D 82 (2010) 074024.

[6] A. D. Martin et al., Eur. Phys. J. C 63 (2009) 189.

[7] A. D. Martin et al., Eur. Phys. J. C 64 (2009) 653.

[8] H1 and ZEUS Collaboration, JHEP 109 (2010) 109.

[9] S. Alekhin et al., Phys. Rev. D 86 (2012) 054009.

[10] CMS Collaboration, [CMS PAS SMP-12-027].

[11] CMS Collaboration, [CMS PAS SMP-12-022].

[12] T. Sjostrand et al., JHEP 0605 (2006) 026.

[13] T. Sjostrand et al., Comput.Phys.Commun. 178 (2008) 852.

[14] M. Bahr et al., Eur.Phys.J. C 58 (2008) 639.

[15] J. Alwall et al., JHEP 1106 (2011) 128.

[16] CMS Collaboration, [hep-ex/1311.5815]. 